\title{
A Time-Domain Morphology and Gradient based Algorithm for ECG Feature Extraction
}

\author{
E. B. Mazomenos*, T. Chen*, A. Acharyya ${ }^{\dagger}$, A. Bhattacharya ${ }^{\ddagger}$, J. Rosengarten $^{\S}$, K. Maharatna* \\ ${ }^{*}$ School of ECS, University of Southampton, Southampton, U.K. \{ebm, tc10g09, km3\}@ecs.soton.ac.uk \\ ${ }^{\dagger}$ EEE Dept., Indian Institute of Technology Guwahati, Guwahati, India. a.acharyya@iitg.ernet.in \\ ${ }^{\ddagger}$ CSE Dept., National Institute of Technology, Durgapur, India. cse.arnab@gmail.com \\ ${ }_{\S}^{\S}$ Southampton University Hospitals NHS Trust, Southampton, UK. james@rosengarten.co.uk
}

\begin{abstract}
A Time Domain Morphology and Gradient (TDMG) based algorithm is presented in this paper for the extraction of all the fiducial time instances from a single PQRST complex. By estimating these characteristic points, all clinically important temporal ECG parameters can be calculated. The proposed algorithm is based on a combination of extrema detection and slope information, with the use of adaptive thresholding to achieve the extraction of 11 time instances. A pre-processing step removes any noise and artefacts from the captured ECG signal. Initially, the position of the R-wave and the QRS-complex boundaries are localized in time. Following, by focusing on the part of the signal that precedes and succeeds the QRScomplex, the remaining fiducial points from the $P$ and $T$ waves are estimated. The initial localisation of the wave boundaries is complimented by amendment steps which are introduced to cater for atypical wave morphologies, indicative of particular heart conditions. The proposed algorithm is evaluated on the QT and PTB databases against medically annotated ECG samples. The results demonstrate the ability of the proposed scheme, to estimate the ECG fiducial points with acceptable accuracy from a single-lead ECG signal. In addition, this investigation reveals the ability of the TDMG algorithm to perform accurately irrespective of the lead chosen, the different disease categories and the sampling frequency of the captured ECG signal.
\end{abstract}

\section{INTRODUCTION}

The most widespread method to observe the electrical activity of the heart over time is the electrocardiogram (ECG). Owing to its non-invasive nature and ease of use, the ECG is utilized in virtually every medical situation which involves the evaluation of the heart's clinical condition. By observing the ECG waveform, physicians can detect possible anomalies in the heart's functionality as well as deduce what type of further medical evaluation is required. Every cardiac cycle (heartbeat) is depicted on the ECG as a sequence of five deflections (the PQRST-complex), each one representing the electrical activity during the different phases of the cardiac cycle .

Since the ECG provides a direct representation of the heart's electrical activity, cardiologists have defined a number of ECG parameters that are used for the evaluation of the heart's condition. These features are either morphological (e.g. Rheight) or temporal (QRS-duration, QT-interval, ST-segment,

This work was supported by E.U. ARTEMIS Joint Undertaking under the Cyclic and person-centric Health management: Integrated appRoach for hOme, mobile and clinical eNvironments - (CHIRON) Project, Grant Agreement \# 2009-1-100228. etc). Fig.1 illustrates a typical PQRST-complex and the relevant ECG features. Evaluation takes place by examining the morphology of the waveform and the value of the clinically important parameters. For example, a prolonged QT-interval is a bio-marker of arrhythmia, while an elongated QRS may be indicative of bundle branch block. Similarly, abnormal wave morphologies (additional peaks, discontinuities) are related to a number of heart conditions (e.g. hypertrophy, myocardial infarction) [1], [2].

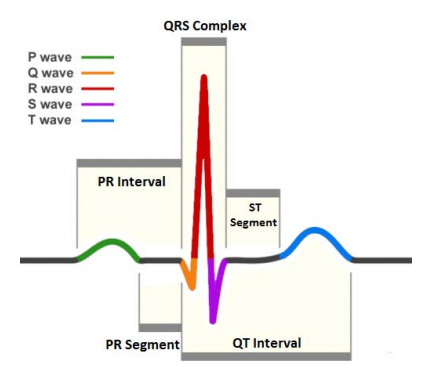

Fig. 1. The PQRST complex. Image reproduced from [3].

The estimation of the aforementioned parameters requires the detection of a total of 11 time instances from the PQRSTcomplex. In details, the onset and offset instances of the Pwave, the QRS-complex and the T-wave, as well as the time instance of the peak of each wave (P,Q,R,S,T) must be extracted. By obtaining this set of 11 time points, all the relevant temporal ECG parameters can be calculated. Additionally, parameters defined between successive heartbeats (e.g. P-P interval, R-R interval) can also be approximated.

A number of techniques and algorithms on ECG feature extraction has been reported on the relevant literature. The majority of these approaches focuses on the detection and time localisation of the QRS-complex. Koöhler et. al. perform a review of the various methodologies and algorithms that have been proposed for QRS detection [4]. The Pan-Tompkins algorithm is one of the first approaches to exploit the steep slope of the QRS complex for detection purposes [5], [6]. Laguna et. al. present a Pan-Tompkins based method for estimating the ECG wave boundaries for multilead ECG systems. The results from different leads are combined to achieve more robust estimation [7]. The use of Wavelet Transform (WT) has also been investigated for feature extraction in ECG signals [8]- 
[10]. WT analysis transforms the ECG signal into its frequency components with their corresponding time localisation [11]. Thus, by investigating the value of WT coefficients at different decomposition levels, the ECG features can be estimated. The "Common Standards for Quantitative Electrocardiography" (CSE) project provided a reference database and also defined the acceptable tolerance limits for automated ECG feature extraction [12], [13] systems.

In this paper, we present the design of a Time-Domain Morphology and Gradient (TDMG) based algorithm for the extraction of the fiducial time instances of the PQRST complex. Different to a number of previous gradient-based approaches, which combine all 15 standard leads in estimating the ECG fiducial points, we focus on a single-lead feature extraction approach. Consequently the TDMG algorithm can be considered in situations where only a limited number of leads is available (e.g. Holter, ambulatory ECG). In addition, the TDMG algorithm also takes into account some atypical ECG morphologies (QRS fragmentation, double hump P,T waves) which are met in a number of abnormal heart rhythms.

The rest of this paper is structured as follows. Section II presents the details of the TDMG algorithm. Experimentation results from applying the TDMG algorithm on ECG signals from two databases are provided in the sequel. Ultimately, Section IV summarizes the paper and discusses ideas for future work.

\section{Proposed TDMG Feature Extraction ALGORITHM}

The proposed technique aims at extracting the 11 parameters of interest as mentioned in Section I from a single PQRSTcomplex. A full PQRST-complex is defined as the sequence of the five composite waves with some isoelectric line segments $(100-130 \mathrm{~ms})$ included at the beginning (before the Pwave) and the end (after the T-wave). The TDMG algorithm is structured as a two-stage process. The first stage pertains to the pre-processing of the sampled PQRST-complex and the second stage is the main feature extraction operation. In essence, the 11 time instances of interest can be grouped into two categories; onset/offset time points and peak time points. The onset/offset time instances are approximated by considering them as inflection points. Due to the morphology of the ECG waves (P,QRS,T), the value of the slope exhibits an abrupt change at the onset/offset time instances of each wave compared to its value at the isoelectric line segments. By capturing this change one can determine the onset/offset time instances. On the other hand, the peak time points are extracted by considering these points as local extrema around their vicinity. Following the standard medical convention, the TDMG algorithm considers that, the Q and S-peak are always negative deflections (local minima) while the R-peak is positive (local maximum). Different to this, the $\mathrm{P}$ and $\mathrm{T}$ peak can be upright (positive deflection), downright (negative deflection) or sometimes biphasic (both positive and negative segments) compared to the isoelectric line. A block diagram of the TDMG algorithm is depicted in Fig. 2.
The two main objectives that the TDMG algorithm attempts to achieve are accuracy and ubiquity. In order for the extracted features to be used for medical evaluation, the desired parameters must be calculated with the maximum possible accuracy. Ubiquity refers to the ability of the algorithm to handle all the intricacies of different heart conditions as these are reflected on the ECG waveform (e.g. ST-elevation, ST-depression, fragmented QRS). In addition, the TDMG algorithm is designed to be independent of the lead used to capture the ECG signal, as well as be able to perform adequately on ECG signals sampled with low sampling frequencies $(100-250 \mathrm{~Hz})$.

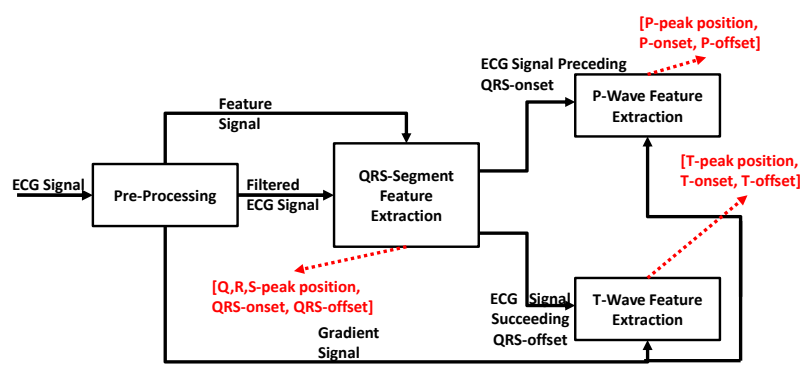

Fig. 2. Block diagram of the TDMG Extraction Algorithm

\section{A. Pre-processing}

The first step of the algorithm pertains to the denoising of the ECG signal from corrupting noise added due to power mains interference, loosely attached electrodes, respiratory and motion artefacts. This is accomplished by passing the ECGsignal through a zero-phase digital band-pass filter, with cutoff frequencies at $0.5 \mathrm{~Hz}$ and at $43 \mathrm{~Hz}$. These are selected based on the fact that the electrical activity of the heart lies within these boundaries [4]. Zero-phase filtering preserves the temporal location of the morphological features present on the filtered signal exactly where those features occur in the unfiltered signal. This step is followed by a smoothing stage where the output of the bandpass filter is passed through a moving average smoothing filter.

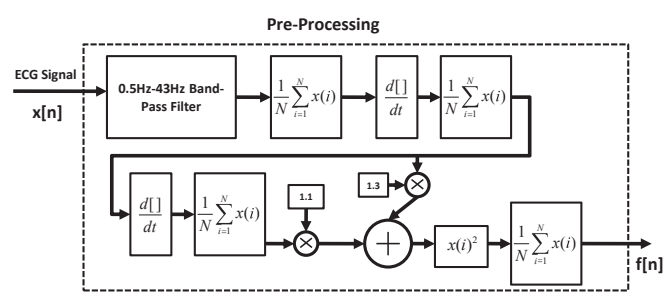

Fig. 3. Pre-Processing Stage. $x[n]$ is the sampled ECG-signal and $f[n]$ is the formulated feature signal.

After the completion of the denoising stage, we proceed to the formulation of the feature signal. Inspired by the PanTompkins algorithm, the feature signal is synthesized as a linear combination of the first and second derivatives of the ECG signal [5]. The derivative signals are calculated with the use of a moving slope filter. This filter operates on a given sliding window and approximates the slope (derivative) 
of that window with the use of a first order model. The smoothing filter is also applied on the derivative signals. The feature signal $f[n]$ is defined as linear combination of the first and second derivative using experimentally verified coefficients; $f[n]=1.3 \operatorname{grad}_{1}[n]+1.1 \operatorname{grad}_{2}[n]$. The feature signal attenuates the $\mathrm{P}$ and T-wave while enhances the QRScomplex. By looking at Fig. 4 it is clear that the boundaries of the QRS complex can be approximated from the feature signal with the use of a threshold policy. The entire pre-processing stage is illustrated in Fig.3.
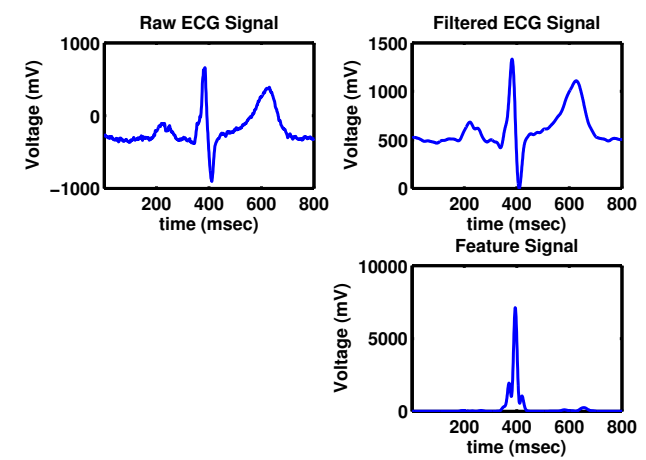

Fig. 4. The outcome of the pre-processing stage.

\section{B. Feature Extraction}

The strategy that is followed in the feature extraction segment of the TDMG algorithm is to first identify the R-peak position, followed by the extraction of the QRS boundaries (QRS-onset and offset) along with the Q-peak and S-peak time instances. Finally, by focusing on the part of the signal that precedes and succeeds the detected QRS-complex, the desired features (onset, offset, peak) of the $\mathrm{P}$ and $\mathrm{T}$-wave, are extracted respectively.

1) Temporal Search Windows: The estimation of the onset/offset time points for each wave (P,QRS,T), is accomplished by investigating the value of the gradient signal (for $\mathrm{P}, \mathrm{T}$ ) or the feature signal (for QRS), within specific time windows around a reference point. The values that are used to define the width of the search window for each point, are based on the experimentation that was carried out during the development of the algorithm and ensure that the point to be estimated, always falls within these windows irrespective of the ECG morphology, the lead, or the sampling frequency of the signal.

2) QRS-complex: Typically the R-wave is the most outstanding feature of the PQRST-complex. However, in a number of leads in the standard 12-lead ECG system, a high negative deflection which corresponds to either the $\mathrm{Q}$ or the $\mathrm{S}$-wave is the most prominent feature. This is caused due to the polarity of the different leads and the direction of the heart's electrical axis. To account for this, we follow a two-stage process in the characterisation of the R-peak time instance.

To begin with, a peak detection algorithm is executed on the PQRST-complex to detect the position in time of all the local maxima. In order for a time instance to be characterised as an extreme point, it must have the highest value in its vicinity and must be preceded by a time point which has a value smaller than a predefined threshold. From the collection of the extreme points, the R-peak time instance is extracted as the local maxima which has the highest gradient value (slope) around it. This is accomplished by summing the value of the gradient signal for a $30 \mathrm{~ms}$ window around the local maxima points and comparing the sums. Nevertheless, in situations where the R-wave height is small, other extreme points (e.g. P-peak, T-peak) may exhibit higher gradient around them, resulting in faulty detection of the R-peak time instance.

For these situations we introduce the second step of the R-peak detection procedure. In this step, we first perform an estimation of the QRS onset and offset positions. The QRS onset and offset time instances are extracted by comparing the value of the feature signal against an adaptive threshold. The maximum point of the feature signal is identified $\left(t_{f \max }\right)$ and used as a reference. The time windows within which the QRS boundaries are searched are $170 \mathrm{~ms}$ wide, defined by the following time instances, $t_{f \max }-200 \mathrm{~ms}$ and $t_{\text {fmax }}-$ $30 m s$ for the QRS-onset and $t_{f \max }+30 \mathrm{~ms}$ and $t_{\text {fmax }}+$ $200 \mathrm{~ms}$ for the QRS-offset. Considering $t_{f \max }$ as reference, the ultimate time instance on the left side window which has a value smaller than a pre-defined threshold is considered to be the QRS-onset, while the first time instance at the right side window that has a value smaller than the same threshold is extracted as the QRS-offset. This procedure is illustrated in Fig. 5. By obtaining the QRS boundaries the amendment on the R-peak position is carried out in the following way.

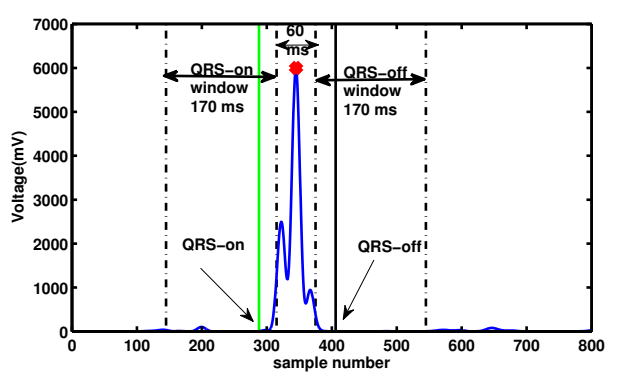

Fig. 5. QRS boundaries extraction from the feature signal.

The extracted R-peak time instance is checked if it falls within the QRS boundaries. If this is not satisfied, the initial approximation of the R-peak position is considered erroneous. The R-peak position is then calculated as the local maxima present within the QRS boundaries. Maxima are calculated with the use of the same peak detection algorithm limited this time in returning at most two maxima for comparison. The complete procedure that is employed for the characterisation of the R-wave is summarized in Proc.1.

Having obtained the final R-peak position, the next step in the feature extraction algorithm includes a refinement of the QRS boundaries and the identification of the time instances for the $\mathrm{Q}$ and $\mathrm{S}$-wave. The refinement on the QRS boundaries is required due to the fact that in ECG signals captured from subjects suffering various diseases, the QRS-complex 


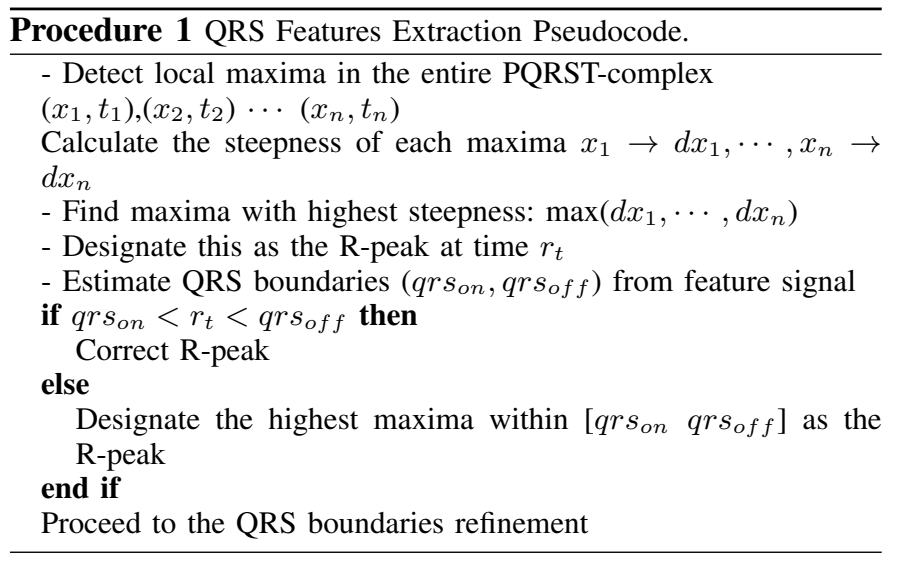

may exhibit fragmented segments. Fragmented QRS shows irregular shapes on the ECG waveform. Examples include, notches, double peaks, and "plateaus". These discontinuities may result in the feature signal having a very small value in these fragmented segments. This value might be erroneously detected as the onset/offset of the QRS-complex. The amendment that is introduced at this stage simply looks at the vicinity of the originally extracted boundaries for values of the feature signal which are 20 times higher than the value of the threshold used to calculate the boundaries. If this is found to be true, this translates to the initial boundaries being erroneous since such high values of the feature signal only exist within the QRS complex. The new boundaries are searched in a smaller window on the left side and right side of the initial boundary for the QRS-onset and QRS-offset respectively. This amending step efficiently deals with any erroneous detections caused from fragmentations or in some cases inadequate signal sampling. An example of this amendment on a fragmented QRS-complex is given in Fig. 6. The "notch" on the S-wave causes the erroneous estimation of the QRS-offset in the first phase and the proposed amendment effectively identifies this and corrects it. The final part of the QRS segment features extraction is devoted to the detection of the Q- and S-peak instances. These are extracted as the local minimum between the QRS-onset and the R-peak and the R-peak and the QRSoffset for the Q-peak and the S-peak respectively.
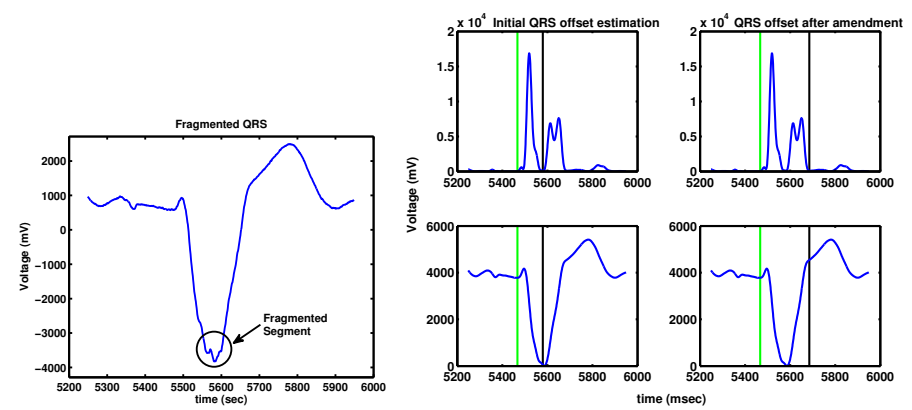

(a) A fragmented QRS-complex (b) QRS offset before and after the amendment

Fig. 6. An example of amending the QRS boundaries due to the presence of a fragmented segment.
3) $P$ and T-wave: The $\mathrm{P}$ and $\mathrm{T}$-waves are curved shaped deflections occurring before and after the QRS-complex. P and $\mathrm{T}$ waves can exhibit either convexity or concavity with respect to the isoelectric line. This is dependent on the lead that captures the ECG signal as well as the heart condition of the subject. In the following, we describe the details of the TDMG algorithm in respect to the P-wave features extraction.

The P-peak is considered to be a local extrema (maximum or minimum) in the portion of the signal that precedes the detected QRS-complex. Since the P-peak can either be a local maximum or minimum point, a peak detection algorithm is tasked with returning the time instances of the local maximum and minimum samples. The decision on which one of these two points is the P-peak is taken by comparing the absolute difference of the two extreme values with the isoelectric line. The value of the isoelectric line is taken as the value of the signal at the QRS-onset which is supposed to be lying on the baseline. The local extrema which demonstrates higher separation from the isoelectric line is considered as the Pwave peak. The time instances of the onset and offset of the $\mathrm{P}$-wave are extracted as the inflection points on the left $(\mathrm{P}$ onset) and right (P-offset) of the P-peak. For the P-onset the portion of the gradient signal $\left(\operatorname{grad}_{1}[n]\right)$ from the beginning up to $35 \mathrm{~ms}$ before the P-peak is checked and the last time instance which has a gradient value smaller than a predefined threshold is identified as the P-onset (see Fig. 7). Similarly, for the Poffset, this investigation starts $25 \mathrm{~ms}$ after the P-peak point and up to $15 \mathrm{~ms}$ before the QRS-onset and the first time point of the gradient, found to be smaller that the same threshold, is extracted as the P-offset.

For the extraction of the T-wave fiducial points, a similar procedure is executed on the portion of the signal that succeeds the detected QRS complex. Because the T-wave typically has a longer duration than the $\mathrm{P}$-wave, longer windows are used around the T-peak instance to partition the signal in the two parts where the algorithm looks for the onset and offset points (see Fig. 7). Moreover, the QRS-offset value is used as the isoelectric reference.

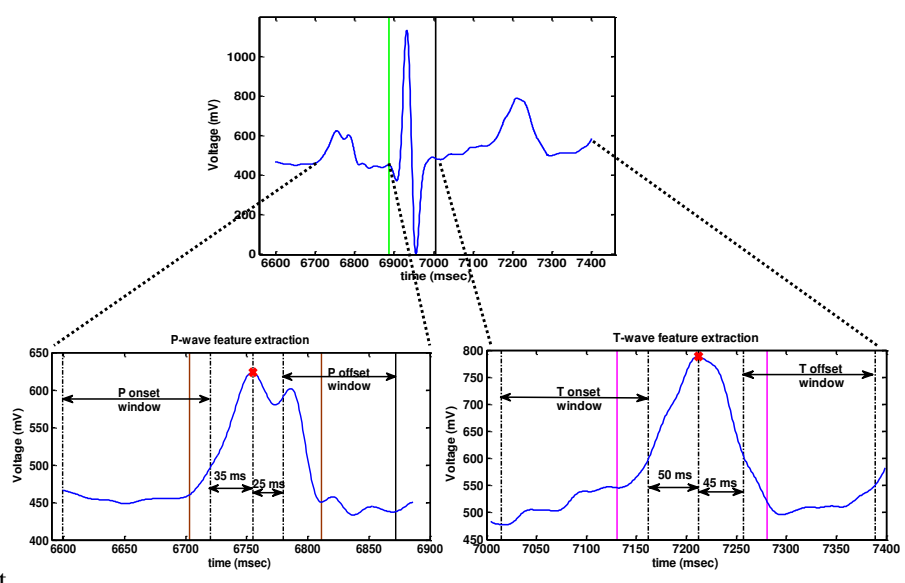

Fig. 7. $\mathrm{P}$ and T-wave feature extraction.

In situations, the $\mathrm{P}$ and/or $\mathrm{T}$-wave may exhibit biphasic 
(both positive and negative deflections) patterns or "double humps" patterns (two sequential convex/concave deflections). Such morphologies are known to be associated with a number of diseases. These peculiar waves may lead the algorithm to erroneous results in the calculation of the onset and offset. To accommodate this, an extra step is carried out for both the $\mathrm{P}$ and $\mathrm{T}$-wave where the value of the ECG signal at the onset and offset points is compared to the value of the signal at the isoelectric line, taken at QRS-onset and QRSoffset for the $\mathrm{P}$-wave and $\mathrm{T}$-wave respectively. These three values must all fall within a certain limit since the three points are supposed to be lying on the isoelectric line. If the difference between these values exceeds a threshold then the onset/offset point is considered to be wrong, thus an amendment takes place where the new onset/offset point is again found using the gradient signal threshold at the part of the signal that precedes/succeeds the initially extracted time point. An example of the refining process being applied on a P-wave which exhibits a "double hump" pattern is illustrated in Fig. 8. Complete results from applying the TDMG algorithm on a PQRST-complex is illustrated in Fig. 9

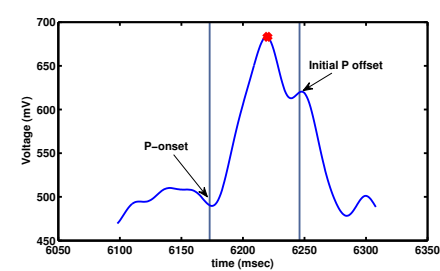

(a) Initial P-wave boundaries

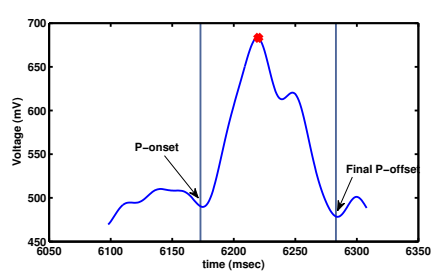

(b) Amended P-wave boundaries
Fig. 8. An example of the P-wave amendment on a "double humped" P-wave.

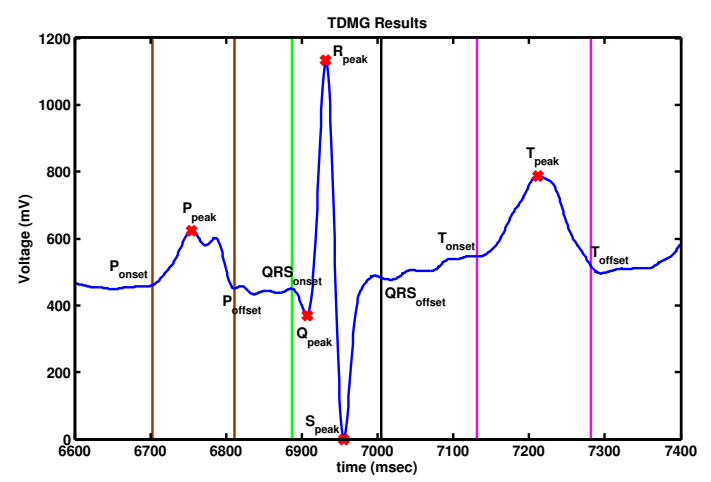

Fig. 9. The estimation of 11 time instances of interest from a single PQRSTcomplex, obtained from the TDMG algorithm.

\section{Adaptive Threshold Selection}

The TDMG algorithm utilizes a number of thresholds for its various operations. One threshold is used to identify the onset and offset of the QRS from the feature signal. Another threshold is used for the $\mathrm{P}$ and $\mathrm{T}$-wave onset and offset extraction. The amendments of the waves boundaries are also based on separate thresholds. These thresholds are introduced in order to ensure the generic applicability of the algorithm, irrespective of the lead that is used and the characteristic ECG morphologies. All the thresholds are defined in a parameterised way, typically being a small percentage of the amplitude range $(\max -\min )$ of the ECG and the featured signal. In addition, the bandpass filter order, the moving average filter window and the time constants used to define the windows, in which the fiducial points are searched, are all defined with respect to the sampling frequency of the ECG signal.

\section{EXPERIMENTATION RESULTS}

The experimental evaluation of the TDMG feature extraction algorithm was carried out using annotated ECG signals from the QT database (QTDB) available at Physionet [14]. From the 105 records of the QTDB, we chose 50 records from the available subgroups and validated our algorithm's with respect to the existing annotations. In addition, ECG signals were extracted from the PTB database (PTBDB) and given to cardiologists for manual annotation. The PTBDB contains standard 15-lead ECG recordings at $1 \mathrm{KHz}$ from 290 subjects from different disease categories. The experts annotated the relevant fiducial points for a single heartbeat from the standard 12-leads for a total of 10 patients covering all the available disease categories, resulting in a pool of 120 ECG heartbeats which was used to evaluate the TDMG algorithm.

The reason for choosing signals from the PTBDB in addition to the extensively used QTDB is to evaluate the performance of the TDMG algorithm on ECG signals from the standard 12-lead system as well as on ECG signals from different disease categories. Our intention is to ascertain the applicability of the TDMG on the standard ECG leads as well as on the various intricate ECG morphologies. In situations where the experts could not identify the presence of a particular wave, predominantly due to the fact that the particular lead does not capture the wave appropriately, the algorithm's results were disregarded.

Table. I, lists results in terms of mean and standard deviation of the error observed between the algorithm's estimation and the medical annotation, from both QTDB and PTBDB signals, as well as the accepted tolerance as it was standardized by the CSE committee [12]. Results from the single-lead threshold detector (TD) method, developed by Jane et al. and applied on the QTDB are also provided here for comparison purposes [15].

The error distribution for each extracted parameter, based on the QTDB experimentation, is illustrated in Fig. 10. It is clear that the error is centered around zero almost for all parameters. The improvement on the performance at the PTBDB is due to the $1 \mathrm{KHz}$ sampling frequency which offers increased temporal resolution compared to the $250 \mathrm{~Hz}$ used in QTDB. This allows for a more accurate estimation, particularly of the onset/offset time instances. Finally, by comparing the results from the TDMG algorithm to the TD, we observe that TDMG outperforms the TD detector in terms of bias in the estimation of the T-wave fiducial points. In the remaining parameters the two methods achieve comparable results in the 


\begin{tabular}{|l|l|l|l|l|l|l|l|l|l|l|}
\hline & Feature & $P_{\text {on }}$ & $P_{\text {peak }}$ & $P_{\text {off }}$ & $Q R S_{\text {on }}$ & $R_{\text {peak }}$ & $Q R S_{\text {off }}$ & $T_{\text {on }}$ & $T_{\text {peak }}$ & $T_{\text {off }}$ \\
\hline \multirow{3}{*}{ QTDB } & no of beats & 1410 & 1413 & 1413 & 1528 & 1528 & 1528 & 464 & 1528 & 1387 \\
\cline { 2 - 11 } & mean (ms) & 0.33 & 7.6 & 11.2 & 4.1 & -5.2 & 5.1 & 12.1 & 2.8 & 5.6 \\
\cline { 2 - 11 } & std (ms) & 21.1 & 15 & 20.8 & 8.7 & 15.6 & 12.4 & 24.6 & 25.3 & 28.6 \\
\hline \multirow{3}{*}{ PTBDB } & no of beats & 114 & 114 & 114 & 120 & 115 & 120 & 118 & 118 & 118 \\
\cline { 2 - 11 } & mean (ms) & 0.2 & -1.2 & -1.5 & -4.1 & -1.4 & 1.3 & -4.1 & 1.1 & -11.9 \\
\cline { 2 - 11 } & std (ms) & 18.7 & 19.2 & 22.4 & 6.1 & 4.5 & 8.7 & 18.8 & 11.3 & 19.8 \\
\hline \multirow{2}{*}{ TD (QTDB) [15] } & mean (ms) & 10.2 & -0.4 & -5.7 & -7.8 & -9.3 & -3.6 & 16 & 23.2 & 18.6 \\
\cline { 2 - 11 } & std (ms) & 14 & 10.9 & 13.5 & 10.8 & 4.4 & 10.7 & 29.8 & 28.2 & 29.7 \\
\hline CSE [12] & std (ms) & 10.2 & - & 12.7 & 6.5 & - & 11.6 & - & - & 30.6 \\
\hline
\end{tabular}

TABLE I

PERFORMANCE RESUlTS OF THE TDMG ALGORITHM ON SIGNALS FROM THE QTDB AND PTBDB AND ACCEPTABLE TOLERANCE LIMITS

sense that the difference in the standard deviation the two algorithms report, is under two samples (i.e. 8ms).
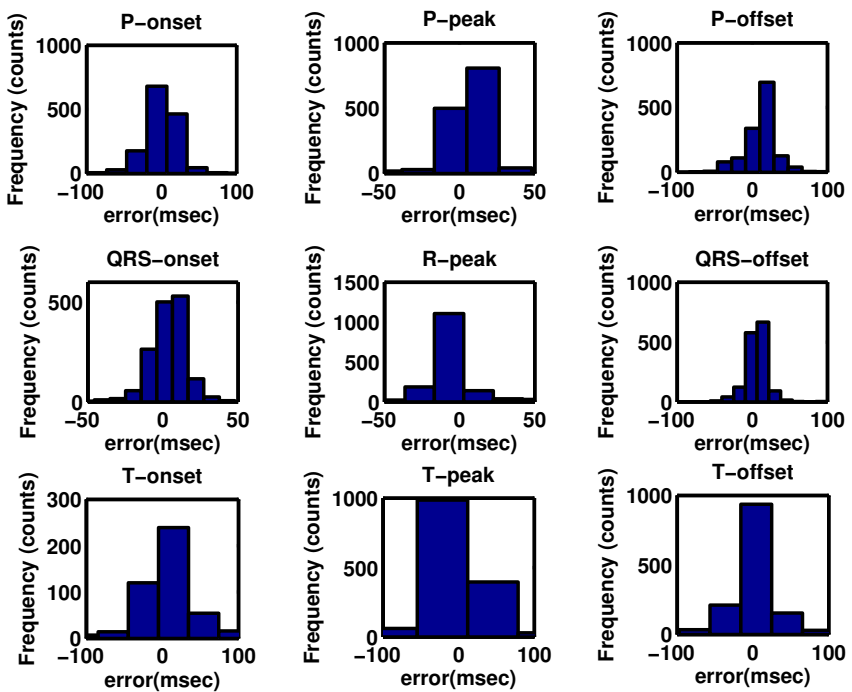

Fig. 10. Error distributions of the extracted parameters from the experimentation on the QTDB.

\section{CONCLUDING REMARKS}

This paper reported the design of the TDMG algorithm for the extraction of all the fiducial points from the ECG waveform. The proposed algorithm is based on the morphological characteristics that the composite waves of the PQRST complex demonstrate. After removing the corrupting noise and artefacts from the ECG signal, the TDMG algorithm initially detects the boundaries of the QRS complex by employing an extended version of the Pan-Tompkins detection method. The proposed extension accounts for fragmented QRS segments, occurring typically in a number of heart conditions. In the sequel the algorithm extracts all the fiducial points from the remaining $\mathrm{P}$ and $\mathrm{T}$ waves by taking advantage of the curvature that these two waves exhibit and considering the onset and offset of these points as inflection points. The TDMG algorithm is evaluated with ECG samples from two databases (QTDB, PTBDB) and the obtained results, apart from the Pwave fiducial points, are either within or very close to the acceptable tolerance limits, particularly in the high resolution signals from PTBDB. Finally, this investigation reveals that the proposed algorithm can be successfully applied in all standard 12 leads, accurately handle intricate ECG morphologies and also perform equally well under signals sampled at different frequencies thanks to an adaptive threshold definition.

Future work involves the investigation of schemes to enhance the robustness of the TDMG algorithm against baseline wandering. This will particularly improve the detection of the $\mathrm{P}$ and T-peaks as this is dependent on the baseline value. We are also working towards the incorporation of a partitioning technique in order to separate a train of ECG heartbeats into individual PQRST-complexes. This will allow the automated analysis of the ECG signal in real-time.

\section{REFERENCES}

[1] R. Baltazar, Basic and Bedside Electrocardiography. Lippincott Williams \& Wilkins, 2009.

[2] T. B. Garcia and N. E. Holtz, 12 Lead ECG: The Art of Interpretation. Jones and Bartlett Learning, 2002.

[3] http://www.skippinghearts.com.

[4] B. U. Köhler, C. Hennig, and R. Orglmeister, "The principles of software qrs detection," IEEE Eng. Med. Biol. Mag, vol. 21, no. 1, pp. 42-57, 2002.

[5] J. Pan and W. J. Tompkins, "A real-time qrs detection algorithm," IEEE Trans Biomed Eng, vol. 32, no. 3, pp. 230-236, mar 1985.

[6] P. S. Hamilton and W. J. Tompkins, "Quantitative investigation of QRS detection rules using the MIT/BIH arrhythmia database." IEEE Trans Biomed Eng, vol. 33, no. 12, pp. 1157-1165, dec 1986.

[7] P. Laguna, R. Jané, and P. Caminal, "Automatic detection of wave boundaries in multilead ecg signals: validation with the cse database," Comput Biomed Res., vol. 27, pp. 45-60, Feb 1994.

[8] S. Mahmoodabadi, A. Ahmadian, M. Abolhasani, M. Eslami, and J. Bidgoli, "Ecg feature extraction based on multiresolution wavelet transform," in IEEE EMBS, Jan 2005, pp. $3902-3905$.

[9] J. Sahambi, S. Tandon, and R. Bhatt, "Using wavelet transforms for ecg characterization. an on-line digital signal processing system," IEEE Eng Med Biol Mag, vol. 16, no. 1, pp. 77 -83, jan.-feb. 1997.

[10] J. Martinez, R. Almeida, S. Olmos, A. Rocha, and P. Laguna, "A wavelet-based ecg delineator: evaluation on standard databases," IEEE Trans Biomed Eng, vol. 51, no. 4, pp. 570-581, Apr 2004.

[11] C. Li, C. Zheng, and C. Tai, "Detection of ecg characteristic points using wavelet transforms," IEEE Trans Biomed Eng, vol. 42, no. 1, pp. $21-28$, jan. 1995.

[12] The CSE working party, "Recommendations for measurement standards in quantitative electrocardiography," Eur Heart J., vol. 6, no. 10, pp. 815-825, Oct 1985.

[13] J.L Willems et al., "Assessment of the performance of electrocardiographic computer programs with the use of a reference database," Circulation, vol. 71, no. 3, pp. 523-5, Mar 1985.

[14] A.L. Goldberger et. al., "Physiobank, physiotoolkit, and physionet components of a new research resource for complex physiologic signals," Circulation, vol. 101, no. 23, 2000 (June 13).

[15] R. Jane, A. Blasi, J. Garcia, and P. Laguna, "Evaluation of an automatic threshold based detector of waveform limits in holter ecg with the qt database," in Comp. Cardio., sep 1997, pp. 295 -298. 